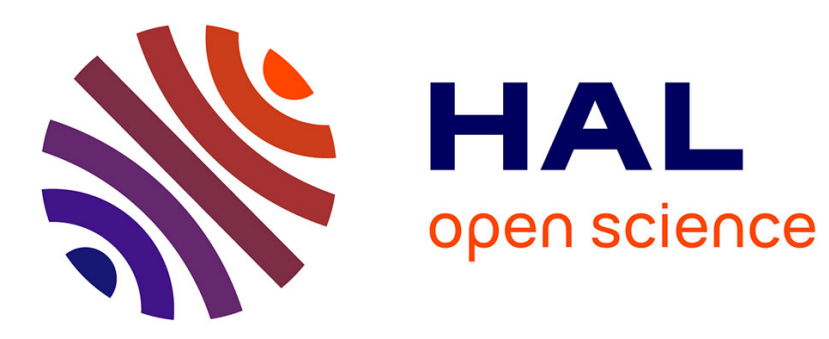

\title{
Coarse-Grained Descriptions
}

Francisco Chinesta, Emmanuelle Abisset-Chavanne

\section{To cite this version:}

Francisco Chinesta, Emmanuelle Abisset-Chavanne. Coarse-Grained Descriptions. A Journey Around the Different Scales Involved in the Description of Matter and Complex Systems A Brief Overview with Special Emphasis on Kinetic Theory Approaches, Springer, pp.41-68, 2018, 10.1007/978-3-31970001-4_3. hal-02794768

\section{HAL Id: hal-02794768 \\ https://hal.science/hal-02794768}

Submitted on 9 Sep 2020

HAL is a multi-disciplinary open access archive for the deposit and dissemination of scientific research documents, whether they are published or not. The documents may come from teaching and research institutions in France or abroad, or from public or private research centers.
L'archive ouverte pluridisciplinaire HAL, est destinée au dépôt et à la diffusion de documents scientifiques de niveau recherche, publiés ou non, émanant des établissements d'enseignement et de recherche français ou étrangers, des laboratoires publics ou privés. 


\title{
Chapter 3 \\ Coarse-grained descriptions
}

\author{
Chinesta, Francisco; Abisset-Chavanne Emmanuelle
}

\subsection{Molecular dynamics}

Molecular dynamics is considered nowadays as a powerful numerical technique able to explore the behavior and structure of the matter at the atomic scale. This technique is more and more used in different fields: computational physics and chemistry, applied mechanics and engineering, ... Molecular dynamics is characterized by: (i) its simplicity from the conceptual point of view and (ii) the impressive CPU time required to perform realistic simulations as well as the too reduced physical systems that can nowadays be analyzed. In any case, this technique seems to be, despite its inherent computational cost, an excellent tool to extract the main behavior of multi-scale models and to analyze localized phenomena that are coupled with the macroscopic scale via the use of appropriate multi-scale techniques or by applying adequate bridges between different zones analyzed at different scales.

When molecular dynamics is applied to a system in equilibrium it allows to determine the temporal evolution of positions, velocities and forces that, using the concepts of statistical mechanics, leads to the calculation of macroscopic properties: elastic constants, surface energy, etc. On the contrary, when the systems evolve off equilibrium, the evolution of these fields (positions, velocities and forces) leads to the calculation of transport properties: thermal conductivity, viscosity, diffusion coefficient, defaults propagation, etc.

In what follows we summarize the main concepts related to the essence and use of this simulation technique, emphasizing its main difficulties that constitute the main research domains of specialists in applied physics, mechanics and mathematics.

The heart of molecular dynamics lies in taking a population of nuclei (without considering explicitly the electrons) whose initial positions and velocities are known or simply assumed. Now, if the inter-atomic potential is known (see section 1.9), its gradient gives the force applied on each nucleus due to the other nuclei as well as to the electronic distribution defined in the whole 3D space (obviously this distribution vanishes far from the region where the nuclei are located). The first trouble arises due to the difficulty (and sometimes impossibility) of solving the Schrödinger equa- 
tion to determine the wavefunction allowing the calculation of that interatomic potential according to the procedure described in section 1.9. For this reason, different potentials have been proposed and used, some are purely phenomenological, others are quantum inspired. Moreover, when one is dealing with molecules involving several kinds of atoms, there are numerous interatomic potentials to be considered, some are related to the strong bonds and others to the weak interactions associated with the Van der Waals effects.

\subsubsection{Some simple examples of pair-wise interatomic potentials}

Hereafter, we are introducing some widely used pairwise potentials. There are many others, some of them concerning three-body and $\mathrm{N}$-body potentials, that can be found in specialized books and papers.

- The hard sphere potential is defined from

$$
V(\mathbf{x} ; \mathbf{X})=\left\{\begin{array}{l}
\infty \text { if }\|\mathbf{x}-\mathbf{X}\| \leq \rho \\
0 \text { if }\|\mathbf{x}-\mathbf{X}\|>\rho
\end{array},\right.
$$

where $\mathbf{X}$ denotes the nucleus position, $\mathbf{x} \in \mathbb{R}^{3}$ and $\rho$ the rigid sphere radius. This kind of potential is only applicable for deriving qualitative behaviors.

- The soft sphere potential is defined by

$$
V(\mathbf{x} ; \mathbf{X})=\varepsilon\left(\frac{\sigma}{\|\mathbf{x}-\mathbf{X}\|}\right)^{n},
$$

where now $\varepsilon, \sigma$ and $n$ are the three coefficients defining the potential. This potential takes into account only the repulsion effects limiting its applicability to some situations.

- The square-well potential is defined by

$$
V=(\mathbf{x} ; \mathbf{X})=\left\{\begin{array}{l}
\infty \quad \text { if }\|\mathbf{x}-\mathbf{X}\| \leq \sigma \\
-\varepsilon \text { if } \sigma<\|\mathbf{x}-\mathbf{X}\| \leq \lambda \sigma, \\
0 \quad \text { if }\|\mathbf{x}-\mathbf{X}\|>\lambda \sigma
\end{array}\right.
$$

with $\lambda>1$, that is the simplest potential taking into account both repulsive and attractive effects. It has been widely used for analyzing fluid properties. There are numerous variations of this potential, the Morse's potential being one of the most popular:

$$
V(\mathbf{x} ; \mathbf{X})=D e^{-2 \alpha\left(\|\mathbf{x}-\mathbf{X}\|-r_{0}\right)}-2 D e^{-\alpha\left(\|\mathbf{x}-\mathbf{X}\|-r_{0}\right)},
$$

where the coefficients $D, \alpha$ and $r_{0}$ depend on the considered material. 
- The Lennard-Jones potential (LJ) is one of the most used in the MD simulations. It allows to consider both attraction and repulsion effects. Among the vast family of $\mathrm{LJ}$ models perhaps the most popular is given by

$$
V(\mathbf{x} ; \mathbf{X})=4 \varepsilon\left[\left(\frac{\sigma}{\|\mathbf{x}-\mathbf{X}\|}\right)^{12}-\left(\frac{\sigma}{\|\mathbf{x}-\mathbf{X}\|}\right)^{6}\right] .
$$

\subsubsection{Integration procedure}

Now, if the force acting on each nucleus is assumed known $\mathbf{F}_{i}, i=1, \ldots, N_{n}$, which as mentioned above is obtained from the gradient of the considered potential, the Newton equation allows to compute the accelerations

$$
\mathbf{a}_{i}=\frac{\mathbf{F}_{i}}{m_{i}}, i=1, \ldots, N_{n},
$$

whose integration allows to update velocities and positions. One of the most widely used integration procedures is the Verlet's algorithm. It is derived from the expansions

$$
\left\{\begin{array}{l}
\mathbf{x}(t+\Delta t)=\mathbf{x}(t)+\left.\sum_{k=1}^{\infty} \frac{1}{k !} \frac{d^{k} \mathbf{x}}{d t^{k}}\right|_{t}(\Delta t)^{k} \\
\mathbf{x}(t-\Delta t)=\mathbf{x}(t)+\left.\sum_{k=1}^{\infty}(-1)^{k} \frac{1}{k !} \frac{d^{k} \mathbf{x}}{d t^{k}}\right|_{t}(\Delta t)^{k}
\end{array}\right.
$$

whose sum results

$$
\mathbf{x}(t+\Delta t)+\mathbf{x}(t-\Delta t)=2 \mathbf{x}(t)+\left.\frac{d^{2} \mathbf{x}}{d t^{2}}\right|_{t}(\Delta t)^{2}+\Theta(\Delta t)^{4},
$$

where $\left.\frac{d^{2} \mathbf{x}}{d t^{2}}\right|_{t}=\mathbf{a}(t)$. This integration scheme, of fourth order, allows to update the nuclei position without using the nuclei velocities. However, these velocities are needed in order to compute the kinetic energy, the velocity distribution, etc. To obtain an expression to compute the nuclei velocities we proceed by subtracting both expansions

$$
\mathbf{x}(t+\Delta t)-\mathbf{x}(t-\Delta t)=\left.2 \frac{d \mathbf{x}}{d t}\right|_{t} \Delta t+\Theta(\Delta t)^{3}
$$

where $\left.\frac{d \mathbf{x}}{d t}\right|_{t}=\mathbf{v}(t)$.

There are numerous integration schemes with different properties of stability, accuracy (energy conservation), implementation simplicity, computing time and computer memory needs, etc. In general the integration strategies are explicit, making possible the use of massive parallel computing platforms. 


\subsubsection{Discussion}

After this brief introduction one could think: where are the difficulties?

Molecular dynamics simulations are confronted, despite its conceptual simplicity, with diverse difficulties of different nature:

- The first and most important comes, as previously indicated, from the impossibility of using an "exact" interaction potential derived from the quantum scale. This situation is particularly delicate when we are dealing with some irregular nuclei distributions as the ones encountered in the neighborhood of defaults in crystals (dislocations, crack-tip, etc.), interfaces between different materials or in zones where different kinds of nuclei coexist.

- The second one comes from the units involved in this kind of simulations: the nuclei displacements are in the nanometric scale, the energies are of the order of the electron-volt $\left(1.610^{-16} \mathrm{~J}\right)$, the time steps are of the order of the picosecond $\left(10^{-15} \mathrm{~s}\right)$. Thus, because of the limits in the computers precision, a change of units is required, which can be easily performed. Moreover, some interatomic potentials in the literature are related to a particular choice of units.

- In molecular dynamics the behavior of atoms and molecules is described in the framework of classical mechanics. Thus, the particles energy variations are continuous. The applicability of MD depends on the validity of this fundamental hypothesis. When we consider crystals at low temperature the quantum effects (implying discontinuous energy variations) are preponderant, and in consequence the matter properties at these temperatures cannot be determined by MD simulations. The use of MD is restricted to temperatures higher than the Debye's temperature. This analysis is in contrast to the vast majority of MD simulations carried out nowadays. In fact, the higher the temperature (kinetic energy), the higher the velocity of particles, requiring shorter time steps in order to ensure the stability of the integration scheme. For this reason, nowadays most of the MD simulations in solid mechanics are carried out at zero degrees Kelvin or at very low temperatures but, as just pointed out, at these temperatures the validity of the computed MD solutions are polluted by the non negligible quantum effects, and it is important to note that many engineering problems implies high temperatures.

- The prescription of boundary conditions is another delicate task. If the analysis is restricted to systems with free boundary conditions, then the MD simulation can be carried out without any particular treatment. In the other case we must consider a system large enough to ensure that in the analyzed region the impact of the free surfaces can be neglected. Another possibility lies in the prescription of periodic boundary conditions, where an atom leaving the system for example through the right boundary is re-injected in the domain through the left boundary. The particles located in the neighborhood of a boundary are influenced by the ones located in the neighborhood of the opposite boundary. The imposition of other boundary conditions is more delicate from both the numerical and the conceptual point of view. For example, what is the meaning of prescribing a displacement on a boundary when the system is not at zero degrees? Each situation 
requires a deep analysis in order to define the best (the most physical) way to prescribe the boundary conditions.

- There are other difficulties related to the transient analysis. Consider a thermal system in equilibrium (where the velocities distribution is in agreement with the Maxwell-Boltzmann distribution). Now, we proceed to heat the system. One possibility lies in increasing suddenly the kinetic energy of each particle. Obviously, even if the resulting velocities define a physical distribution, the system remains off equilibrium because the partition between kinetic and potential energies is not the appropriate one. For this reason we must proceed to relax the system that evolves from this initial state to the equilibrium one. Other (more physical) possibility lies in incorporating a large enough ambient region around the analyzed system (the so-called thermostat), whose particles are initially in equilibrium at the highest temperature. Now, both regions (the system and the ambient) interact, and the system initiates its heating process that reaches its equilibrium some time latter. The final state of both evolutions is the same, but the time required to reach it depends on the technique used to induce the heating. The first transient is purely numerical whereas the second one is more physical allowing the identification of some transport coefficients (e.g. thermal conductivity).

- Finally the CPU time continues to be the main limitation of MD simulations. The strongest handicap is related to the necessity of considering at each time step and for each particle the influence of all the others particles. Thus, the integration method seems to scale with the square of the number of particles. Even if some computing time savings can be introduced in the neighbors search, the extremely small time steps and the extremely large number of particles required to describe real scenarios, limit considerably the range of applicability of this kind of simulations, that has been accepted to be nowadays of the order of some cubic micrometers, even when the systems are considered at very low, and then non-physical, temperatures (close to zero ${ }^{\circ} \mathrm{K}$ ). We can notice that, despite the impressive advances in the computational capabilities, the high performance computing and the use of massive parallel computing platforms, the state-of-the-art does not allow the treatment of macroscopic systems encountered in practical applications of physics, chemistry and engineering.

In conclusion, MD is conceptually very easy to understand, not too difficult to implement, but extremely expensive in terms of computing time and resources. Its discrete nature implies simplicity, but at the same time the technique becomes computationally expensive to be efficiently applied to analyze large macroscopic systems.

\subsubsection{Recovering macroscopic behaviors}

In this section we are illustrating the emergence of macroscopic behaviors from the extremely detailed atomic description, justifying the interest of conducting molecu- 
lar dynamics simulations despite the too small scales (in space and time) nowadays attainable.

When solving the Schrödinger equation for a simple atom, for example the Lithium one, composed of three electrons, the ground-state defines a problem in 9 dimensions and we obtain, in agreement with the Pauli exclusion principle, the electronic distribution illustrated in Fig. 3.1. The first two electrons occupy the socalled $s$-orbital, an almost spherical shell around the atom nucleus, while the third one is distributed in one of the three available $p$-orbitals (the shapes of these orbitals are depicted in Fig. 3.2).
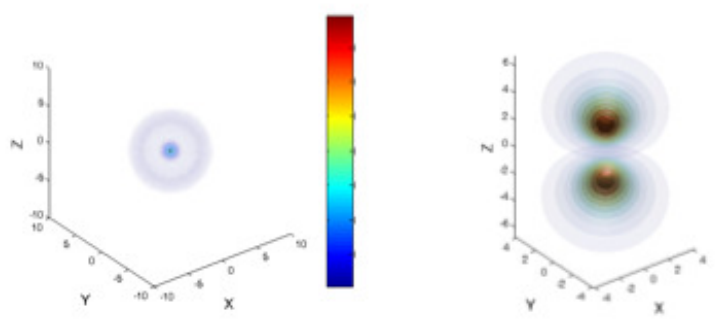

Fig. 3.1 Lithium atom: Electronic distribution

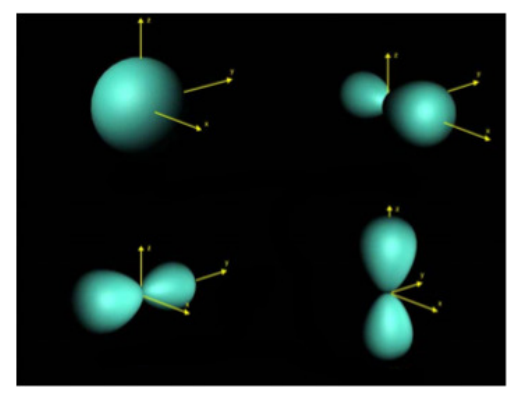

Fig. 3.2 Shape of the $s$ and $p$ electronic orbitals

The same procedure can be applied to more complex systems composed of different atoms in order to investigate the electronic distribution in simple molecules. As discussed in the previous chapter when considering the Schrödinger equation, in general we assume fixed the nuclei positions and compute the associated electronic distribution, that consequently will depend parametrically on the nuclei positions. The electronic distribution and in particular its ground state is related to the lowest energy, that as just commented will depends on the chosen location of the nuclei.

When considering a simple molecule composed of two atoms, we can compute the energy associated with the ground state as a function of the relative distance between both nuclei. By assuming both atoms on the $x$-coordinate axis, the first 
located at $X$ (e.g. $X=0)$ and the second one placed at $X+r(r$ when $X=0)$, with $r \in$ $\mathbb{R}^{+}$, the ground-state energy (the so-called two-particles potential) will depend on the inter-nuclei distance $r, V(r)$, and as discussed above, it is in general adequately represented by the Lennard-Jones potential. A typical representation is depicted in Fig. 3.3 where different facts can be noticed:

- When both particles move apart, $r \rightarrow \infty$, both the potential and its gradient vanish. Since the inter-particle force acts in the opposite direction to the potential gradient, being $\left.\nabla V(r)\right|_{r \rightarrow \infty} \approx 0$, the resulting force vanishes and both particles become free.

- When particles approach one to the other the solution of the Schödinger equation results in an electronic distribution that concentrates in the region in between both nuclei. Thus, electrons become a sort of glue that compensate the mutual repulsion of both positively charged nuclei, ensuring the molecule stability. The potential gradient for $r>r_{\min }$ is positive and the resulting force negative. The attractive force tends to approach the test nucleus to the one assumed located at the origin $X=0$ and kept at rest.

- When the nuclei are too close one to the other, the electronic distribution that results from the Schrödinger solution leaves the region in between both nuclei and then both positively charged nuclei becomes directly exposed one to the other. A repulsion appears (negative potential gradient, then positive force) that tends to move apart both nuclei.

- The equilibrium distance ( $r_{\min }$ in Fig. 3.3), the one for which the potential gradient vanishes, defined the reference interatomic distance.

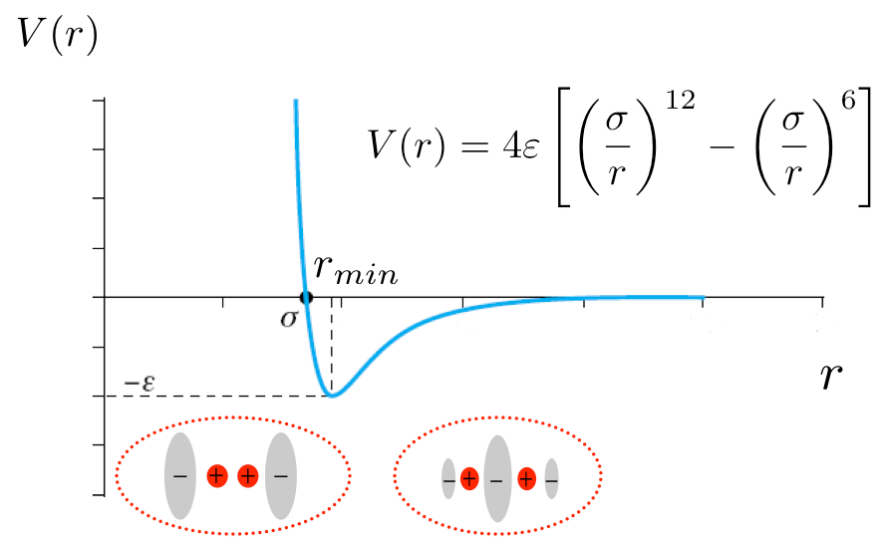

Fig. 3.3 Two-particles potential $V(r)$ evolution with the inter particle distance $r$

When the molecule contains just the energy involved in the potential (blue curve), no remaining part exists related to the kinetic energy. However, if an extra-energy $\Delta E$ is communicated to the system as illustrated in Fig. 3.4, the energy gap $E-$ 
$V(r)$ represents the kinetic energy available in the molecule. Thus, if we assume the nucleus located at $X=0$ at rest, the one initially located at $r_{\min }$ must move in the allowed region, the one defined by $E-V(r) \geq 0$ (red arrow in Fig. 3.4). Obviously, in the periodic movement, the two limits of the interval are defined by $E-V(r)=0$ and in both points the kinetic energy vanishes. On the contrary when $r=r_{\min }$ the kinetic energy $E-V\left(r_{\min }\right)$ reaches its highest value. It is a simple view of the fact of atoms are continuously vibrating when there is an excess of energy.

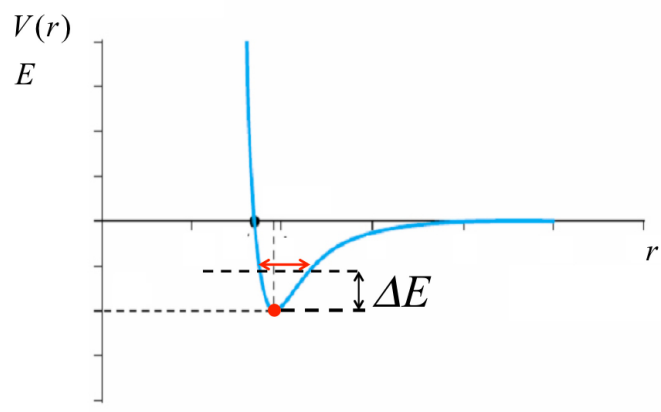

Fig. 3.4 Communicating a kinetic energy to a molecular system

Another important fact that Fig. 3.3 reflects is the dilatation properties. As the potential curve around its minimum at $r=r_{\min }$ is non-symmetric it implies that, when heating, the energy level $E$ varies and with it the vibration amplitude as illustrated in Fig. 3.5. Thus, the average position $\bar{r}=\frac{1}{T} \int_{0}^{T} r(t) d t$ (with $T$ the vibration period) increases as the energy gap increases, explaining usual dilatation in materials.

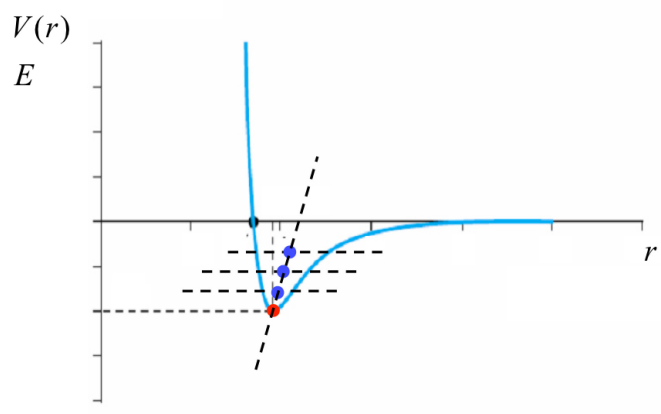

Fig. 3.5 Explaining dilatation in materials

Finally, because the potential gradient results in the interatomic forces, considering a virtual surface within a solid composed of many nuclei and calculating the resultant of all the inter-particles forces crossing that surface, forces that depend 
on the relative distance between each pair of nuclei, by projecting on the different coordinate axes and dividing by the surface, allows to define the Cauchy stress, as illustrated in Fig. 3.6. Moreover, if the potential $V(r)$ around its minimum at $r_{\min }$ is parabolic, the related force becomes linear, since its slope (the potential curvature at $\left.r_{\min }\right)$ is the elastic modulus.

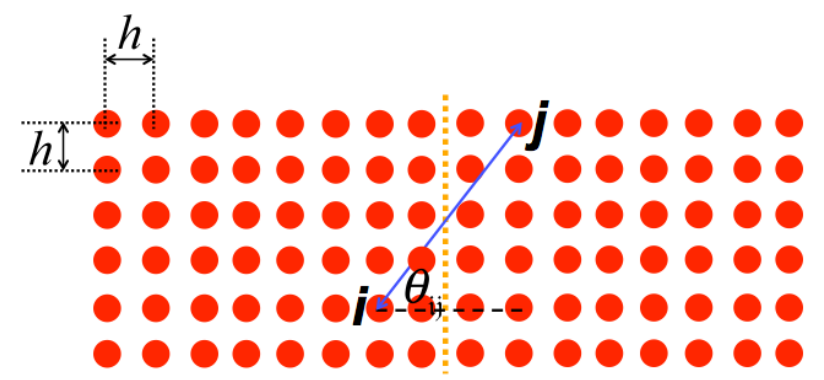

Fig. 3.6 Forces applying between pairs of nuclei at each side of the considered surface ara calculated and then projected.

On the other hand, the maximum force that a bond can resists is less than the magnitude of the maximum potential gradient in the region $r \geq r_{\text {min }}$.

Finally, we are describing the emergence of transport properties. For that purpose, we consider a circular ring composed of two regions, one at high temperature and the other (much larger) at lower temperature, as illustrated in Fig. 3.7 (left). At the initial time we assume Maxwellian velocity distributions in both regions, as depicted in Fig. 3.7 (right).
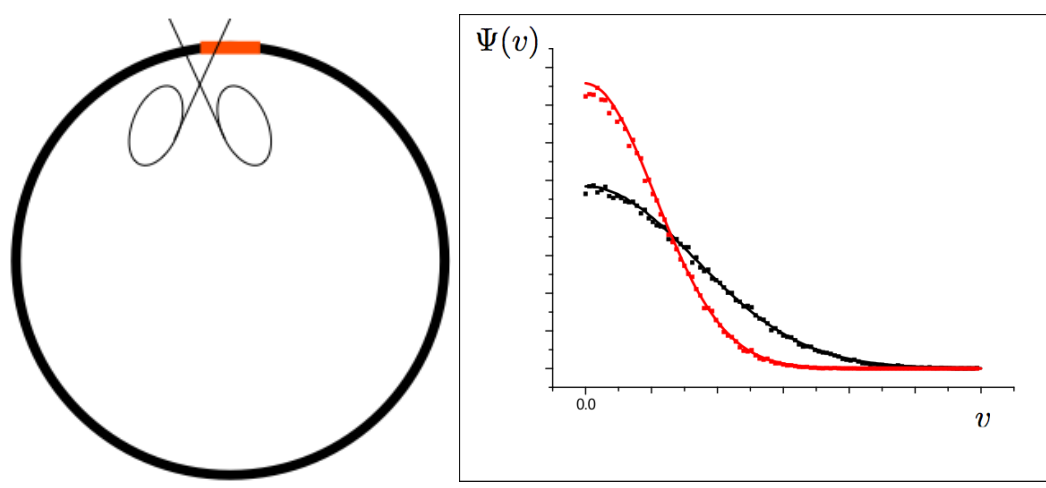

Fig. 3.7 Unbounded circular ring composed of two regions (left) with initial Maxwellian velocity distributions (right). The scissors indicates that the domain will be represented unfolded in Fig. 3.8

The Verlet integration scheme is applied, and as expected, because the atoms in the coldest region close to the warmest one are pushed with higher intensity from the 
heated side, they increase their velocity (temperature) while the ones in the warmest region pushing them, lose energy, as depicted in figure 3.8. Thus, the heat propagates from the warmest region to the coldest one, and again molecular dynamics allows to explain the macroscopic Fourier law. The speed of propagation determines the material thermal diffusivity.

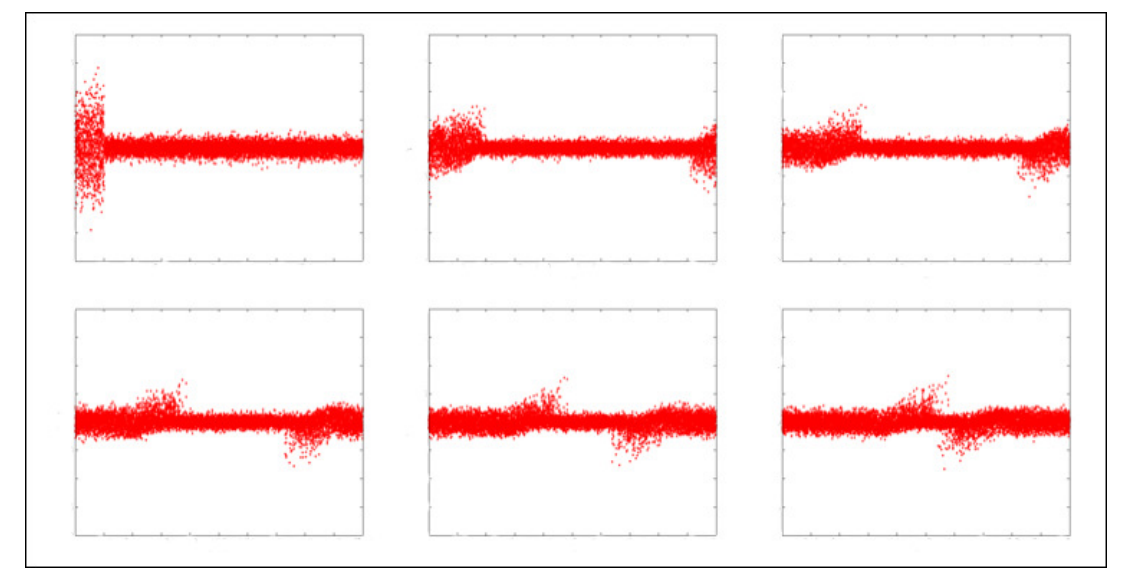

Fig. 3.8 Heat propagation by conduction in the ring depicted in Fig. 3.7 using an unfolded representation. Thermal diffusivity emerges from molecular dynamics simulations.

\subsubsection{Molecular dynamics-continuum mechanics bridging techniques}

The above mentioned difficulties to perform fully molecular dynamics simulations motivated the proposal of hybrid techniques that apply MD in the regions where the unknown field varies in a non-uniform way (molecular dynamics model) and a standard finite element approximation in those regions where the unknown field variation can be considered as uniform (continuous model). The main questions concerned by these bridging strategies concern: (i) the kinematics representations in both models; (ii) the transfer conditions on the MD and continuous models interface; and (iii) the macroscopic constitutive equation to be employed in the continuous model.

Different alternatives exist, and the construction of such bridges is nowadays one of the most actives topics in computational mechanics. The spurious reflection of the high frequency parts of waves is one of the main issues. We would like only to mention two families of bridging techniques, giving some key references:

1. The quasi-continuum method proposed by Tadmor and Ortiz can be applied for establishing bridges between MD and continuum models [47]. It links atomistic 
and continuum models through the use of finite element to reduce the full set of atomistic degrees of freedom. Thus, in the regions where the solution evolution is non-uniform the full atomistic description is retained. In those regions where the solution evolves uniformly it is possible to select a (reduced) number of representative atoms to describe the kinematics (via the finite element interpolation) and the energy of the body. This observation suggests a division of the representative atoms into two classes: (i) nonlocal atoms whose energies are computed by an explicit consideration of all their neighbors according to the MD practice; and (ii) local atoms whose energies are computed from the local deformation gradients using the Cauchy-Born (which states that the atoms in the deformed crystal will move to positions dictated by the existing gradients of displacements). Thus, instead of using of phenomenological constitutive equation in the continuum model, such as the Hooke's law, this technique uses atomistic calculations to inform the energetic statement of the continuum mechanics variational principle. Special treatments must be employed to avoid the "ghost" forces appearing in the transition zones.

2. Ben Dhia proposed in 1998 [9] a superposition technique able to define efficient and accurate bridges in multiscale, multimodel and multiphysics (the Arlequin method). These ideas have been recently adopted by numerous teams that have emphasized in particular the excellent ability of this approach for coupling MD and continuum models [50,54]. The main idea is to define an overlapping region in which models (MD and continuum) coexist. The energy in the fully MD and continuum regions is defined without ambiguity, however the Arlequin approach defines the energy in the overlapping region from a linear combination of the ones related to MD and continuum models. At a certain point, the weights of both energies depend on the position of that point. When the point (inside the overlapping region) approaches the fully MD region, the weight related to the MD energy approaches one and the weight related to the continuum energy vanishes. When the point approaches the fully continuum domain the inverse tendencies are found, and when the point is located inside the overlapping region the weights are computed according to the distance to each region under the constraint of an unit sum of both. Thus, the energy can be perfectly defined everywhere. The kinematics is also defined without ambiguity on the MD and continuum regions, and a weak equality of the MD and continuum displacements is enforced in the overlapping region.

\subsubsection{Coarse-grained molecular dynamics: DPD and MPCD}

Conventional MD provides too much detail of the actual motion of the molecules of a fluid. If one is interested in hydrodynamic behavior one can look at a more coarse-grained level. 


\subsubsection{Dissipative Particle Dynamics - DPD}

The particles in DPD are not real molecules but a sort of molecule clusters called 'fluid particle". By introducing dissipation into a molecular-dynamics simulation, one expects to observe hydrodynamic behavior with a considerably smaller number of particles, thus reducing the computational effort. In the DPD not only the number of particles is conserved but also the total momentum of the system.

For a fluid particle with momentum $\mathbf{p}_{i}$, its time derivative is equal to the net force applying on it

$$
\dot{\mathbf{p}}_{i}=\sum_{j \neq i}\left(\mathbf{F}_{i j}^{C}+\mathbf{F}_{i j}^{D}+\mathbf{F}_{i j}^{R}\right),
$$

where $\bullet^{C}, \bullet^{D}$ and $\bullet^{R}$ refer to the conservative, dissipative and random forces respectively. Because of the fluctuation-dissipation theorem the last two forces must apply jointly.

The conservative force comes from the gradient of a potential (as it was the case in molecular dynamics). Galilean invariance requires that both the dissipative and random forces depend on the relative positions and velocities, $\mathbf{r}_{i j}=\mathbf{r}_{i}-\mathbf{r}_{j}$ and $\mathbf{v}_{i j}=$ $\mathbf{v}_{i}-\mathbf{v}_{j}$ respectively, where $\mathbf{r}_{i}$ and $\mathbf{v}_{i}$ are the position and velocity of particle $i$.

The simplest form of these forces guaranteeing these hypotheses is

$$
\mathbf{F}_{i j}^{D}=-\gamma \omega_{D}\left(r_{i j}\right)\left(\mathbf{e}_{i j} \cdot \mathbf{v}_{i j}\right) \mathbf{e}_{i j}
$$

and

$$
\mathbf{F}_{i j}^{R}=\sigma \omega_{R}\left(r_{i j}\right) \zeta_{i j} \mathbf{e}_{i j}
$$

where $r_{i j}=\left\|\mathbf{r}_{i}-\mathbf{r}_{j}\right\|, \mathbf{e}_{i j}=\frac{\mathbf{r}_{i}-\mathbf{r}_{j}}{r_{i j}}$ and $\zeta_{i j}$ are Gaussian white-noise random variables such that $\zeta_{i j}=\zeta_{j i}$ for ensuring the momentum conservation, with the stochastic properties

$$
\left\{\begin{array}{l}
\left\langle\zeta_{i j}(t)\right\rangle=0 \\
\left\langle\zeta_{i j}(t) \zeta_{k l}\left(t^{\prime}\right)\right\rangle=\left(\delta_{i k} \delta_{j l}+\delta_{i l} \delta_{j k}\right) \delta\left(t-t^{\prime}\right)
\end{array},\right.
$$

and where $\omega_{D}$ and $\omega_{R}$ define the interaction horizon for dissipation and random forces.

Even if many simulations considered $\omega_{D}=\omega_{R}$, in [29] it was proved that the verification of the fluctuation-dissipation theorem requires to consider $\omega_{R}(r)=$ $\sqrt{\omega_{D}(r)}$.

\subsubsection{Multi-Particle Collision Dynamics - MPCD}

MPCD consists of two stages, streaming and collision, ensuring the conservation of particles, mass and linear momentum, as well as a sufficient degree of isotropy to reproduce hydrodynamic behavior. Its computational simplicity and possibility of considering highly parallel implementations explains the growing interest within the scientific community. 
The system is composed by $N$ particles, each of them located at position $\mathbf{r}_{i}(t)$ and having a velocity $\mathbf{v}_{i}(t)$. We assume that collision only occur at discrete timeintervals $\tau$. Thus, the new position of particle $i$ at time $t+\tau$ reads

$$
\mathbf{r}_{i}^{*}(t+\tau)=\mathbf{r}_{i}(t)+\mathbf{v}_{i} \tau
$$

Now, for considering collisions we proceed as follows. The volume $V$ is divided into $N_{c}$ cells, $\mathscr{C}_{\alpha}, \alpha=1, \cdots, N_{c}$, each of them containing instantaneously $N_{\alpha}$ particles. We can define the $\alpha$-cell center of mass velocity $\mathbf{V}_{\alpha}$ from

$$
\mathbf{V}_{\alpha}=\frac{1}{N_{\alpha}} \sum_{i \in \mathscr{C}_{\alpha}} \mathbf{v}_{i}
$$

Then, a random rotation is assigned to each cell $\Omega_{\alpha}$ and apply on all the particles that it contains, that results in the post-collision velocities given by

$$
\mathbf{v}_{i}^{*}=\mathbf{V}_{\alpha}+\Omega_{\alpha}\left(\mathbf{v}_{i}-\mathbf{V}_{\alpha}\right), \quad \forall i \in \mathscr{C}_{\alpha} .
$$

MPCD has the following properties:

- Mass conservation is trivially ensured;

- Linear momentum is conserved at each cell. The proof proceeds as follows

$$
\sum_{i \in \mathscr{C}_{\alpha}} m \mathbf{v}_{i}^{*}=\sum_{i \in \mathscr{C}_{\alpha}} m\left(\mathbf{V}_{\alpha}+\Omega_{\alpha}\left(\mathbf{v}_{i}-\mathbf{V}_{\alpha}\right)\right),
$$

since the rotation is the same for all the particles into the cell $\mathscr{C}_{\alpha}$

$$
\begin{gathered}
\sum_{i \in \mathscr{C}_{\alpha}} m \Omega_{\alpha}\left(\mathbf{v}_{i}-\mathbf{V}_{\alpha}\right)=m \Omega_{\alpha} \sum_{i \in \mathscr{C}_{\alpha}}\left(\mathbf{v}_{i}-\mathbf{V}_{\alpha}\right)= \\
m \Omega_{\alpha}\left(\left(\sum_{i \in \mathscr{C}_{\alpha}} \mathbf{v}_{i}\right)-N_{\alpha} \mathbf{V}_{\alpha}\right)=0
\end{gathered}
$$

it finally results

$$
\sum_{i \in \mathscr{C}_{\alpha}} m \mathbf{v}_{i}^{*}=\sum_{i \in \mathscr{C}_{\alpha}} m \mathbf{v}_{\alpha}=\sum_{i \in \mathscr{C}_{\alpha}} m \mathbf{v}_{i}
$$

- Finally, energy conservation follows from

$$
\sum_{i \in \mathscr{C}_{\alpha}} m\left(\mathbf{v}_{i}^{*}\right)^{2}=\sum_{i \in \mathscr{C}_{\alpha}} m\left(\mathbf{V}_{\alpha}+\Omega_{\alpha}\left(\mathbf{v}_{i}-\mathbf{V}_{\alpha}\right)\right)^{2} .
$$

By developing the squared term

$$
\begin{gathered}
\left(\mathbf{V}_{\alpha}+\Omega_{\alpha}\left(\mathbf{v}_{i}-\mathbf{V}_{\alpha}\right)\right)^{2}=\left(\mathbf{V}_{\alpha}^{T}+\left(\mathbf{v}_{i}-\mathbf{V}_{\alpha}\right)^{T} \Omega_{\alpha}^{T}\right)\left(\mathbf{V}_{\alpha}+\Omega_{\alpha}\left(\mathbf{v}_{i}-\mathbf{V}_{\alpha}\right)\right)= \\
\mathbf{V}_{\alpha}^{T} \mathbf{V}_{\alpha}+\left(\mathbf{v}_{i}-\mathbf{V}_{\alpha}\right)^{T} \Omega_{\alpha}^{T} \Omega_{\alpha}\left(\mathbf{v}_{i}-\mathbf{V}_{\alpha}\right)+ \\
\left(\mathbf{v}_{i}-\mathbf{V}_{\alpha}\right)^{T} \Omega_{\alpha}^{T} \mathbf{V}_{\alpha}+\mathbf{V}_{\alpha}^{T} \Omega_{\alpha}\left(\mathbf{v}_{i}-\mathbf{V}_{\alpha}\right)
\end{gathered}
$$


that taking into account the orthogonality of $\Omega_{\alpha}, \Omega_{\alpha}^{T} \Omega_{\alpha}=\mathbf{I}$, leads to

$$
\begin{gathered}
\left(\mathbf{V}_{\alpha}+\Omega_{\alpha}\left(\mathbf{v}_{i}-\mathbf{V}_{\alpha}\right)\right)^{2}= \\
\mathbf{V}_{\alpha}^{T} \mathbf{V}_{\alpha}+\left(\mathbf{v}_{i}-\mathbf{V}_{\alpha}\right)^{T}\left(\mathbf{v}_{i}-\mathbf{V}_{\alpha}\right)+\left(\mathbf{v}_{i}-\mathbf{V}_{\alpha}\right)^{T} \Omega_{\alpha}^{T} \mathbf{V}_{\alpha}+\mathbf{V}_{\alpha}^{T} \Omega_{\alpha}\left(\mathbf{v}_{i}-\mathbf{V}_{\alpha}\right)
\end{gathered}
$$

Now, when applying the sum, and taking into account (3.18) the last two terms in Eq. (3.22) vanish, and then it results

$$
\sum_{i \in \mathscr{C}_{\alpha}} m\left(\mathbf{v}_{i}^{*}\right)^{2}=\sum_{i \in \mathscr{C}_{\alpha}} m\left(\mathbf{V}_{\alpha}^{T} \mathbf{V}_{\alpha}+\mathbf{v}_{i}^{T} \mathbf{v}_{i}-\mathbf{V}_{\alpha}^{T} \mathbf{v}_{i}-\mathbf{v}_{i}^{T} \mathbf{V}_{\alpha}+\mathbf{V}_{\alpha}^{T} \mathbf{V}_{\alpha}\right),
$$

that taking into account

$$
\sum_{i \in \mathscr{C}_{\alpha}} \mathbf{V}_{\alpha}^{T} \mathbf{v}_{i}=N_{\alpha} \mathbf{V}_{\alpha}^{T} \mathbf{V}_{\alpha}
$$

and

$$
\sum_{i \in \mathscr{C}_{\alpha}} \mathbf{V}_{\alpha}^{T} \mathbf{V}_{\alpha}=N_{\alpha} \mathbf{V}_{\alpha}^{T} \mathbf{V}_{\alpha}
$$

it finally results

$$
\sum_{i \in \mathscr{C}_{\alpha}} m\left(\mathbf{v}_{i}^{*}\right)^{2}=\sum_{i \in \mathscr{C}_{\alpha}} m \mathbf{v}_{i}^{2}
$$

When different type of particles are involved, each one is considered with its mass. When coupling fluid and rigid or deformable objects it suffices to use the same collision operator; the streaming must be applied to the fluid particles and finally the kinematics of particles composing the object will be derived from the Hamiltonian defining the object behavior.

If one uses a fixed grid to define the collision cells, one breaks the system symmetry under Galilei transformations. Imagine a fluid in a situation where the mean free path during the time-step is substantially smaller than the collision cell size $\Delta x$. Then, given a particular collision cell, the set of particles in that cell at time $t$ is going to contain mostly the same members that at the next time step (collision time). Statistically, the states of those particles are therefore going to be correlated over a time large compared to the streaming time-step.

However, if one superimposes a global, fixed and non-zero velocity on the entire system, the correlation time changes in general, since now, the sets of particles in the cell at different times may share less members. This means that the statistical properties of a system depend on the observer inertial frame, breaking Galilean symmetry.

This deficiency of broken Galilean symmetry can be eliminated by independently sampling three random numbers defining the vector $\mathbf{S}$ and shifting either the entire collision cell grid by $\mathbf{S}$ with reference to its fixed position in the previous scenario, or equivalently, by shifting the positions of all MPC particles by $-\mathbf{S}$. 


\subsection{Brownian dynamics: A step towards coarse-grained models}

Looking for significant computing time savings, different coarse-grained models have been proposed and successfully used. One of these approaches is the Brownian dynamics (BD) simulation and some variants of it.

We are summarizing the main ideas related to this framework by considering a simple scenario, the one reveled by Robert Brown in 1827. Robert Brown observed that small and light particles immersed in a fluid show a kind of erratic trajectory in the microscopic scale, even if there is an average movement tendency induced by gravity or the fluid drift. This fact is today justified by the fact that atoms are in constant movement (since their kinetic energy is proportional to the temperature) exploring all the possible movement directions. This constitutes the foundations of the kinetic theory of gases proposed by Maxwell and Boltzmann. Due to this pseudo-erratic movement sometimes the fluid atoms impact the suspended particles inducing their change of quantity of movement and then the change in their direction of motion.

From the previous analysis one could expect that the only possibility for simulating this kind of scenario lies in taking into account all the atoms existing in the fluid as well as all the small test particles immersed inside and proceeding in the context of MD. However, the number of particles involved is too large with respect to the nowadays computer availabilities. Remember that the state of the art in MD simulation allows to treat systems in the order of some cubic micrometers. For this reason one could imagine the removal of all the fluid atoms but retaining their effects on the particles of interest, that is, the impacts statistics.

\subsubsection{The Langevin equation}

In the situation just described, the motion equation for each one of the $N$ particles in the suspension reads according to classical mechanics

$$
m \frac{d^{2} x_{i}}{d t^{2}}+\xi \frac{d x_{i}}{d t}=\Re_{i}(t) ; \quad i=1, \ldots, N .
$$

For the sake of simplicity we assume 1D particle motions (the extension to 3D is straightforward) whose positions are defined by the coordinate $x_{i}$. In Eq. (3.27), also known as Langevin's equation, $m$ is the particles mass, $\xi$ the friction coefficient (that in the case of the Stokes drag for spherical particles reads $\xi=6 \pi r \eta$, with $r$ the sphere radius and $\eta$ the fluid viscosity) and $\Re_{i}$ the impact to which particle $i$ is exposed from the ambient atoms. In this equation $m \frac{d^{2} x_{i}}{d t^{2}}$ represents the inertia term, $\xi \frac{d x_{i}}{d t}$ the viscous resistance and $\Re_{i}(t)$ the external forces originated by the atoms impacts. The viscous force is proportional to the difference between the particle velocity and the unperturbed fluid velocity at the position of the particle $v_{f}\left(x_{i}\right)$, implying in general a term in the form: $\xi\left(\frac{d x_{i}}{d t}-v_{f}\left(x_{i}\right)\right)$ that simplifies to the viscous 
term considered in Eq. (3.27) when the unperturbed fluid remains at rest. Eq. (3.27) represents the balance of forces applied on each particle, whose integration only needs the specification of the impact forces. Obviously, this statistical distribution becomes well defined as soon as both its mean value and its variance (or the associated standard deviation) are given. In an isotropic medium one could expect that the average of all the impacts is zero. Moreover, as these impacts are uncorrelated, we can equate time and ensemble averages, and write

$$
\langle\Re(t)\rangle=\frac{1}{N} \sum_{i=1}^{N} \Re_{i}(t)=0 .
$$

Now, if we have access to the standard deviation of the impact statistics the motion equation (3.27) will be perfectly defined making possible its numerical integration. For this purpose, we are introducing a highly valuable result in statistics, the central limit theorem that states that the sum $Y_{n}(t)$ of any $n$ random variables $y_{i}(t)$, converges to a normal distribution whose mean value and variance result the sum of mean values $\left\langle y_{i}\right\rangle$ and variances $\left(\Delta y_{i}\right)^{2}$ of the random variables involved, i.e.

$$
Y_{n}(t) \equiv \sum_{i=1}^{i=n} y_{i}(t) \quad \overrightarrow{n \rightarrow \infty} \mathscr{N}\left(\sum_{i=1}^{i=n}\left\langle y_{i}\right\rangle, \sum_{i=1}^{i=n}\left(\Delta y_{i}\right)^{2}\right) .
$$

As the time elapsed between two consecutive impacts $\delta t$ is much lower than the simulation time step $\Delta t$ employed to integrate the motion equation (3.27), we can define the action $B_{\Delta t} \equiv \int_{t}^{t+\Delta t} \frac{\Re(t)}{m} d t$ that using the fact that $\delta t \ll \Delta t$ as well as the central limit theorem results

$$
\int_{t}^{t+\Delta t} \frac{\Re(t)}{m} d t=\sum_{j=1}^{p} \frac{\Re\left(t_{j}\right)}{m} \delta t \rightarrow \mathscr{N}(0, q \Delta t),
$$

where $p=\frac{\Delta t}{\delta t} \gg 1$ justifying that the variance of the resulting normal distribution will be proportional to the time step $\Delta t$. In what follows we are trying to identify the value of factor $q$ in Eq. (3.30).

The integration of the Langevin equation (3.27) (see [51] for details) leads to the equilibrium velocity distribution

$$
W(v, t \rightarrow \infty)=\sqrt{\frac{\xi}{m \pi q}} e^{-\frac{\xi v^{2}}{m q}},
$$

that must coincide with the equilibrium distribution associated with the canonical ensemble (based on the equipartition theorem), implying

$$
q=\frac{2 \xi K_{b} T}{m^{2}}
$$


where $K_{b}$ is the Boltzmann constant and $T$ the temperature. Expression (3.32) states that the strength of the Brownian force is related to the viscous force (fluctuationdissipation relation).

In conclusion, the simulation of the Langevin equation, or its more general expression involving an external potential $V(x)$

$$
m \frac{d^{2} x_{i}}{d t^{2}}+\xi \frac{d x_{i}}{d t}+\frac{d V}{d x}=\Re_{i}(t) ; \quad i=1, \ldots, N,
$$

only requires for a population of particles large enough, the use of an appropriate numerical integration scheme and the consideration of a normal random variable to model the impacts

$$
\frac{\Re_{\Delta t}}{m}=\mathscr{N}\left(0, \frac{2 \xi K_{b} T}{m^{2}} \Delta t\right) .
$$

\subsubsection{From diffusion to anomalous diffusion}

In his pioneering work Einstein assumed the increment of the particle position $\Delta$ in the unbounded one-dimensional axis $x$ as a random variable, with a probability density given by $\phi(\Delta)$. The particles balance can be expressed from both

$$
\rho(x, t+\tau)=\rho(x, t)+\frac{\partial \rho}{\partial t} \tau+\Theta\left(\tau^{2}\right),
$$

and

$$
\rho(x, t+\tau)=\int_{\mathbb{R}} \rho(x+\Delta, t) \phi(\Delta) d \Delta .
$$

Developing $\rho(x+\Delta, t)$

$$
\rho(x+\Delta, t)=\rho(x, t)+\frac{\partial \rho}{\partial x} \Delta+\frac{1}{2} \frac{\partial^{2} \rho}{\partial x^{2}} \Delta^{2}+\Theta\left(\Delta^{3}\right)
$$

that injected into the right-hand side of Eq. (3.35) and taking into account the normality and expected symmetry

$$
\left\{\begin{array}{l}
\int_{\mathbb{R}} \phi(\Delta) d \Delta=1 \\
\int_{\mathbb{R}} \Delta \phi(\Delta) d \Delta=0
\end{array},\right.
$$

leads, after equating Eqs. (3.35) and (3.36), to

$$
\rho(x, t)+\frac{\partial \rho}{\partial t} \tau=\rho(x, t)+\frac{1}{2} \frac{\partial^{2} \rho}{\partial x^{2}} \int_{\mathbb{R}} \Delta^{2} \phi(\Delta) d \Delta,
$$

or

$$
\frac{\partial \rho}{\partial t} \tau=\frac{1}{2} \frac{\partial^{2} \rho}{\partial x^{2}} \int_{\mathbb{R}} \Delta^{2} \phi(\Delta) d \Delta
$$


Now, by defining the diffusion coefficient $D$ from

$$
D=\frac{1}{2 \tau} \int_{\mathbb{R}} \Delta^{2} \phi(\Delta) d \Delta,
$$

the particles balance, also known as diffusion equation, is given by

$$
\frac{\partial \rho}{\partial t}=D \frac{\partial^{2} \rho}{\partial x^{2}} .
$$

The integration of this equation assuming that all the particles are localized at the origin at the initial time, $\rho(x, t=0)=\delta(x)$, leads

$$
\rho(x, t)=\frac{1}{\sqrt{4 \pi D t}} e^{-\frac{x^{2}}{4 D t}},
$$

whose second order moment (variance) scales with the time

$$
\left\langle x^{2}\right\rangle=2 D t,
$$

that is, the mean squared displacement scales with the elapsed time $t$, and the diffusion coefficient $D$.

By applying the Fourier transform with respect to the space coordinate to the diffusion equation (3.42), and denoting by $\rho(k, t)$ the Fourier transform of the particles density $\rho(x, t)$, i.e. $\rho(k, t)=\mathscr{F}[\rho(x, t)]$ with $k$ the so-called wave number, it results

$$
\frac{\partial \rho(k, t)}{\partial t}=-D k^{2} \rho(k, t),
$$

that has important physical consequences. A general function contains many frequencies, and as it can be appreciated in the previous equation, the lower the wavelength (higher values of $k$ ) the faster its relaxation. Thus, highest frequencies are expected to disappear very fast compared to the lowest ones. Moreover, in the case of negative diffusion (encountered in phase separation) highest frequencies grow faster than the lowest ones and if no mechanism limits the growing process it diverges.

\subsubsection{The diffusion equation from a random walk perspective}

In this section we revisit the derivation of the diffusion equation from a random walk perspective. Again for the sake of simplicity we restrict our discussion to the $1 \mathrm{D}$ case, with the $x$-axis equipped with a grid of size $\Delta x$. We assume that in a discrete time step $\Delta t$ the test particle is assumed to jump to one of its nearest neighbour sites, with random direction. Such a process can be modeled by the master equation that writes at the site $j$

$$
W_{j}(t+\Delta t)=\frac{1}{2} W_{j+1}(t)+\frac{1}{2} W_{j-1}(t),
$$


where $W_{j}(t)$ is the probability of having the particle at site $j$ at time $t$ and the prefactor $1 / 2$ accounts for the direction isotropy of the jumps.

Now, usual developments can be performed:

$$
\left\{\begin{array}{l}
W_{i}(t+\Delta t)=W_{j}(t)+\left.\frac{\partial W_{j}(t)}{\partial t}\right|_{t} \Delta t+\Theta\left(\Delta t^{2}\right) \\
W_{j+1}(t)=W_{j}(t)+\left.\frac{\partial W(t)}{\partial x}\right|_{j} \Delta x+\left.\frac{1}{2} \frac{\partial^{2} W(t)}{\partial x^{2}}\right|_{j} \Delta x+\Theta\left(\Delta x^{3}\right), \\
W_{j-1}(t)=W_{j}(t)-\left.\frac{\partial W(t)}{\partial x}\right|_{j} \Delta x+\left.\frac{1}{2} \frac{\partial^{2} W(t)}{\partial x^{2}}\right|_{j} \Delta x-\Theta\left(\Delta x^{3}\right)
\end{array}\right.
$$

that injected into Eq. (3.46) yields

$$
\frac{\partial W}{\partial t}=D \frac{\partial^{2} W}{\partial x^{2}}
$$

with $D$ defined in the limit of $\Delta x \rightarrow 0$ and $\Delta t \rightarrow 0$ by

$$
D=\frac{\Delta x^{2}}{2 \Delta t}
$$

that leads to the diffusion equation derived in the previous section.

\subsubsection{Anomalous diffusion and the continuous time random walk}

In complex fluids, micro-rheological experiments often exhibit anomalous subdiffusion or sticky diffusion, in which the mean square displacement of Brownian tracer particles is found to scale as $\left\langle x^{2}\right\rangle \propto t^{\alpha}, 0<\alpha<1$ (see [33] and the references therein). In these cases, the use of non-integer derivatives can constitute an appealing alternative as it allows one to correctly reproduce the observed physical behaviour while keeping the model as simple as possible. Moreover, from a physical point of view, the use of non-integer derivatives introduces a degree of non-locality that seems in agreement with the intrinsic nature of the physical system.

In order to move towards anomalous diffusion, we consider continuous time random walks (CTRW) that will lead to a fractional diffusion equation in the same manner as standard random walks led to the usual diffusion equation.

Before developing that equation we are summarizing the most salient concepts related to fractional derivatives.

\section{Fractional derivatives}

There are many books on fractional calculus and fractional differential equations (e.g. $[44,36])$. We summarize here the main concepts needed to understand the developments carried out below.

We start with the formula usually attributed to Cauchy for evaluating the $n$-th integration, $n \in \mathbb{N}$, of a function $f(t)$ : 


$$
(t-\tau)^{n-1} f(\tau) d \tau
$$

$$
J^{n} f(t):=\int \cdots \int f(\tau) d \tau=\frac{1}{(n-1) !} \int_{0}^{t}
$$

This can be rewritten as

$$
J^{n} f(t)=\frac{1}{\Gamma(n)} \int_{0}^{t}(t-\tau)^{n-1} f(\tau) d \tau,
$$

where $\Gamma(n)=(n-1)$ ! is the gamma function. The latter being in fact defined for any real value $\alpha \in \mathbb{R}$, we can define the fractional integral from

$$
J^{\alpha} f(t):=\frac{1}{\Gamma(\alpha)} \int_{0}^{t}(t-\tau)^{\alpha-1} f(\tau) d \tau
$$

Now, if we consider the fractional derivative of order $\alpha$ and we select an integer $m \in \mathbb{N}$ such that $m-1<\alpha<m$, then it suffices to consider an integer $m$-order derivative combined with a $(m-\alpha)$ fractional integral (a sketch is depicted in Fig. 3.9). Obviously, we could take the derivative of the integral or the integral of the derivative, resulting in the left and right-hand definitions of the fractional derivative usually denoted by $D^{\alpha} f(t)$ and $D_{*}^{\alpha} f(t)$ respectively

$$
D^{\alpha} f(t)= \begin{cases}\frac{d^{m}}{d t^{m}}\left(\frac{1}{\Gamma(m-\alpha)} \int_{0}^{t} \frac{f(t)}{(t-\tau)^{\alpha+1-m}} d \tau\right), & m-1<\alpha<m \\ \frac{d^{m} f(t)}{d t^{m}}, & \alpha=m\end{cases}
$$

and

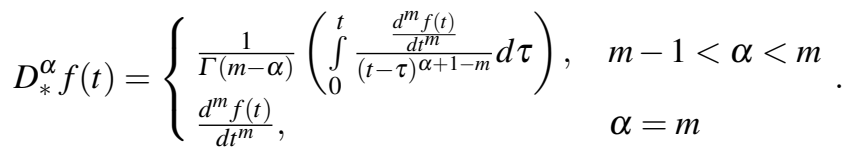

Because these approaches to the fractional derivative began with an expression for the repeated integration of a function, one could consider a similar approach for the derivative. This was the route considered by Grunwald and Letnikov - GL - that defined the so-called 'differintegral' that leads to the fractional counterpart of the usual finite differences.

The Fourier transform of a fractional derivative of order $\alpha$ reads $\mathscr{F}(g(t) ; \omega)=$ $(i \omega)^{\alpha} \mathscr{G}(\omega)$, and analogously when considering the Laplace transform.

\section{The factional diffusion equation}

We are now going to see how continuous time random walks (CTRW) leads to a fractional diffusion equation.

CTRW is based on the fact that the length jump and the waiting time between two successive jumps are given by the probability density function - pdf $-\psi(x, t)$. From it we can define the jump length pdf $\lambda(x)$ from the marginal probability 


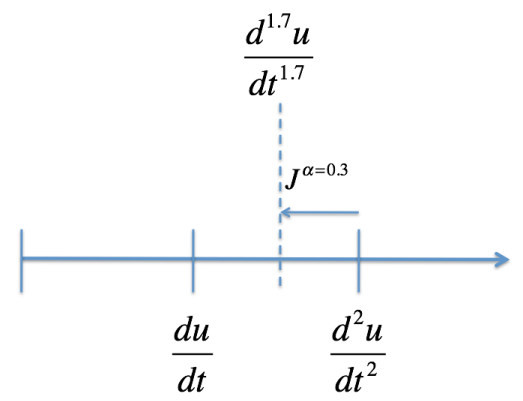

Fig. 3.9 Schematic cartoon of the derivative of order 1.7

$$
\lambda(x)=\int_{0}^{\infty} \psi(x, t) d t
$$

and the waiting time pdf by

$$
\omega(t)=\int_{-\infty}^{\infty} \psi(x, t) d x
$$

Thus, CTRW can be characterized by the characteristic waiting time $T$

$$
T=\int_{0}^{\infty} t \omega(t) d t
$$

and the jump length variance $\Sigma^{2}$

$$
\Sigma^{2}=\int_{-\infty}^{\infty} x^{2} \lambda(x) d x
$$

that can be, both, finite or diverging.

Now, we write the master equation

$$
\eta(x, t)=\int_{-\infty}^{\infty} d s \int_{0}^{t} \eta(s, \tau) \psi(x-s, t-\tau) d \tau+g(x) \delta(t)
$$

where the last term accounts for the initial condition, with a distribution $g(x)$ at the initial time $t=0$. When all the probability concentrates at the origin of coordinates $g(x)=\delta(x)$, with $\delta(\bullet)$ the Dirac mass. The previous master equation relates the 
pdf $\eta(x, t)$ of just arriving at location $x$ at time $t$, with the ones of being arrived at location $s$ at time $\tau$.

Thus, the probability of being at position $x$ at time $t, W(x, t)$, is given by

$$
W(x, t)=\int_{0}^{t} \eta(x, \tau) \Upsilon(t-\tau) d \tau,
$$

where $r(t-\tau)$ represents the probability that no jump takes place in the time interval $[t-\tau, t]$, and can be calculated from

$$
\Upsilon(t)=1-\int_{0}^{t} \omega(\tau) d \tau,
$$

whose Laplace transform readss

$$
\Upsilon(u)=\frac{1-\omega(u)}{u} .
$$

Eq. (3.60) can be also written as

$$
W(x, t)=\int_{0}^{t} \eta(x, t-\tau) \Upsilon(\tau) d \tau .
$$

By Introducing Eq. (3.59) into Eq. (3.63) it results

$$
\begin{gathered}
W(x, t)=\int_{0}^{t}\left(\int_{-\infty}^{\infty} d s \int_{0}^{t-\tau} \eta(s, \theta) \psi(x-s, t-\tau-\theta) d \theta+g(x) \delta(\tau)\right) \Upsilon(\tau) d \tau= \\
\int_{0}^{t}\left(\int_{-\infty}^{\infty} d s \int_{0}^{t-\tau} \eta(s, \theta) \psi(x-s, t-\tau-\theta) d \theta\right) \Upsilon(\tau) d \tau+g(x) \Upsilon(t)= \\
\int_{-\infty}^{\infty} d s \int_{0}^{t} d \tau\left(\int_{0}^{t-\tau} \eta(s, \theta) \psi(x-s, t-\tau-\theta) d \theta\right) \Upsilon(\tau)+g(x) \Upsilon(t) .
\end{gathered}
$$

On the other hand,

$$
\begin{gathered}
\int_{-\infty}^{\infty} d s \int_{0}^{t} d \tau W(s, \tau) \psi(x-s, t-\tau)= \\
\int_{-\infty}^{\infty} d s \int_{0}^{t} d \tau\left(\int_{0}^{\tau} \eta(\tau-\theta) \Upsilon(\theta) d \theta\right) \psi(x-s, t-\tau)=
\end{gathered}
$$




$$
\int_{-\infty}^{\infty} d s \int_{0}^{t} d \theta\left(\int_{\theta}^{t} \eta(\tau-\theta) \psi(x-s, t-\tau) d \tau\right) \Upsilon(\theta) .
$$

By defining $\tau-\theta=u$, the previous integral (3.65) reads

$$
\begin{aligned}
& \int_{-\infty}^{\infty} d s \int_{0}^{t} d \theta\left(\int_{\theta}^{t} \eta(\tau-\theta) \psi(x-s, t-\tau) d \tau\right) \Upsilon(\theta)= \\
& \int_{-\infty}^{\infty} d s \int_{0}^{t} d \theta\left(\int_{0}^{t-\theta} \eta(u) \psi(x-s, t-\theta-u) d u\right) \Upsilon(\theta),
\end{aligned}
$$

that coincides with the integral part of expression (3.64). Thus injecting Eq. (3.66) into Eq. (3.64) we obtain the master equation

$$
W(x, t)=\int_{-\infty}^{\infty} d s \int_{0}^{t} d \tau W(s, \tau) \psi(x-s, t-\tau)+g(x) \Upsilon(t),
$$

that by applying both Laplace and Fourier transforms reduces to

$$
W(k, u)=W(k, u) \psi(k, u)+\Upsilon(u) W_{0}(k),
$$

with $W_{0}(x)$ the Fourier transform of the initial condition, with $W_{0}(x)=1$ when $g(x)=\delta(x)$. Fom Eq. (3.68) it results

$$
W(k, u)=\frac{\Upsilon(u)}{1-\psi(k, u)} W_{0}(k),
$$

that taking into account Eq. (3.62) reads

$$
W(k, u)=\frac{W_{0}(k)}{1-\psi(k, u)} \frac{1-\omega(u)}{u},
$$

that represents the searched solution in the Fourier/Laplace spaces.

\subsubsection{Long rests versus long jumps: subdiffusion and Levy flights}

We consider now different cases of CTRW with decoupled jump pdf, i.e. $\psi(x, t)=$ $\omega(t) \lambda(x)$. If both the characteristic waiting time $T$ and the jump length variance $\Sigma^{2}$ are finite, the long-time limit corresponds to a Brownian motion.

If we consider a Poissonian waiting time pdf

$$
\omega(t)=\frac{1}{\tau} e^{-\frac{t}{\tau}}
$$

with $T=\tau$, and a Gaussian jump length pdf $\lambda(x)$ 


$$
\lambda(x)=\frac{1}{\sqrt{4 \pi \sigma^{2}}} e^{-\frac{x^{2}}{4 \sigma^{2}}},
$$

leading to $\Sigma^{2}=2 \sigma^{2}$, then the Laplace and Fourier transforms of $\omega(t)$ and $\lambda(x)$ respectively read

$$
\left\{\begin{array}{l}
\omega(u)=1-u \tau+\Theta\left(\tau^{2}\right) \\
\lambda(k)=1-\sigma^{2} k^{2}+\Theta\left(k^{4}\right)
\end{array} .\right.
$$

Thus, the jump pdf can be approximated at the lowest orders from

$$
\psi(k, u) \approx 1-u \tau-\sigma^{2} k^{2} .
$$

In fact, as proven in [37], any pair of pdfs leading to finite $T$ and $\Sigma^{2}$ leads at lower orders to the same results, and then in the long-time limit.

By introducing the expansions (3.73) and (3.74) into Eq. (3.70), in results

$$
W(k, u)=\frac{W_{0}(k)}{u+\mathscr{K} k^{2}},
$$

with $\mathscr{K}=\frac{\sigma^{2}}{\tau}$.

Now, from the Fourier and Laplace transforms properties, and in particular

$$
\mathscr{F}\left\{\frac{\partial^{2} W(x, t)}{\partial x^{2}}\right\}=-k^{2} W(k, t),
$$

and

$$
\mathscr{L}\left\{\frac{\partial W(x, t)}{\partial t}\right\}=u W(x, u)-W_{0}(x),
$$

reorganizing Eq. (3.75)

$$
u W(k, u)-W_{0}(k)+\mathscr{K} k^{2} W(k, u)=0,
$$

we can identify the diffusion equation

$$
\frac{\partial W}{\partial t}=\mathscr{K} \frac{\partial^{2} W}{\partial x^{2}}
$$

\section{Long rest and subdiffusion}

We consider a long-tailed waiting time pdf whose characteristic time $T$ diverges,

$$
\omega(t)=A\left(\frac{\tau}{t}\right)^{1+\alpha},
$$

with $0<\alpha<1$, whose Laplace transform writes

$$
\omega(u) \approx 1-(u \tau)^{\alpha},
$$


that injected into Eq. (3.70) leads to

$$
W(k, u)=\frac{\frac{W_{0}(k)}{u}}{1+\mathscr{K}_{\alpha} u^{-\alpha} k^{2}},
$$

with $\mathscr{K}_{\alpha}=\frac{\sigma^{2}}{\tau^{\alpha}}$.

Taking into account the Laplace transform applied to fractional derivatives, in particular

$$
\mathscr{L}\left\{D^{-p} W(x, t)\right\}=u^{-p} W(x, u),
$$

for $p \geq 0$, it results

$$
W(x, t)-W_{0}(x)=D^{-\alpha}\left(\mathscr{K}_{\alpha} \frac{\partial^{2} W(x, t)}{\partial x^{2}}\right) .
$$

By taking the time derivative to Eq. (3.84) it results

$$
\frac{\partial W(x, t)}{\partial t}=D^{1-\alpha}\left(\mathscr{K}_{\alpha} \frac{\partial^{2} W(x, t)}{\partial x^{2}}\right)
$$

however a more valuable expression consists in removing the time derivatives in the right-hand side of Eq. (3.84). For that purpose it suffices to apply a fractional derivative $D^{\alpha}$ to both members. It is important to notice that the Riemann-Liouville fractional derivative of a constant does not vanish, in fact it is given by $D^{\alpha} 1=$ $\frac{1}{\Gamma(1-\alpha)} t^{-\alpha}$. Thus, from Eq. (3.84) it results

$$
D^{\alpha} W(x, t)-\frac{t^{-\alpha}}{\Gamma(1-\alpha)} W_{0}(x)=\mathscr{K}_{\alpha} \frac{\partial^{2} W(x, t)}{\partial x^{2}} .
$$

The mean squared displacement can be calculated from Eq. (3.75), using the relation

$$
\left\langle x^{2}\right\rangle=\lim _{k \rightarrow 0}\left\{-\frac{\partial^{2} W(k, u)}{\partial k^{2}}\right\}
$$

and performing the subsequent Laplace inversion that results in

$$
\left\langle x^{2}\right\rangle=\frac{2 \mathscr{K}_{\alpha}}{\Gamma(1+\alpha)} t^{\alpha}
$$

leading to subdiffusion for $\alpha<1$ and to standard diffusion as son as $\alpha=1$.

\section{Long jumps and Levy flights}

Finally, instead of considering a diverging waiting time, we considers a diverging jump length variance $\Sigma^{2}$. For the waiting time we consider the Poissonian pdf and for the jump length distribution we consider the Lévy distribution that in the Fourier 
space is expressed from

$$
\lambda(k)=e^{-\sigma^{\mu}|k|^{\mu}} \approx 1-\sigma^{\mu}|k|^{\mu},
$$

for $1<\mu<2$, that corresponds to the asymptotic behavior $\lambda(x) \approx A_{\mu} \sigma^{-\mu}|k|^{-1-\mu}$. In the present case, and as previously indicated, the mean squared displacement diverges.

By injecting expression (3.89) into Eq. (3.70) it results

$$
W(k, u)=\frac{1}{u+\mathscr{K}_{\mu}|k|^{\mu}},
$$

with $\mathscr{K}_{\mu}=\frac{\sigma^{\mu}}{\tau}$. The Laplace and Fourier inversion lead to the fractional equation

$$
\frac{\partial W(x, t)}{\partial t}=\mathscr{K}_{\mu} D_{-\infty}^{\mu} W(x, t),
$$

where $D_{-\infty}^{\mu}$ denotes the fractional derivative previously defined but when now the lower integration limit is taken at $-\infty$.

Thus, subdiffusion is related to fractional time derivative whereas superdiffusion places the fractional derivative in the space derivatives. 